\title{
Validity of Upper Gastro-Intestinal Endoscopic Screening in HCV Cirrhotic Patients Awaiting Antiviral Therapy
}

\author{
Salem Y. Mohamed1', Bassim A. Gaballah'1, Mohamed H. Emara2* \\ ${ }^{1}$ Internal Medicine Department, Faculty of Medicine, Zagazig University, Zagazig, Egypt \\ ${ }^{2}$ Tropical Medicine Department, Faculty of Medicine, Zagazig University, Zagazig, Egypt \\ Email: "emara20007@yahoo.com
}

Received 19 October 2015; accepted 20 December 2015; published 23 December 2015

Copyright (C) 2015 by authors and Scientific Research Publishing Inc.

This work is licensed under the Creative Commons Attribution International License (CC BY).

http://creativecommons.org/licenses/by/4.0/

(c) (i) Open Access

\section{Abstract}

Background and Aim: The Egyptian Ministry of Health initiated a nationwide HCV treatment program with the newly developed oral antiviral therapies and formulated national guidelines for treatment allocation which gave favor for patients with advanced fibrosis and early cirrhosis. One of the recommendations for treatment was upper Gastro-intestinal (GIT) endoscopy. This study aimed at estimating the prevalence of varices among those patients and judging the validity of this national recommendation. Methods: This study was carried out at gastrointestinal endoscopy units, Zagazig University Hospitals through the year 2014. The epidemiologic, clinical features and endoscopic findings of patients undergoing preparation for HCV therapy were analyzed. Endoscopic classifications of esophageal and gastric varices were carried out after the Italian liver cirrhosis project and Sarin's classification respectively. Results: Totally 1143 patients performed upper GIT endoscopy as preparation for HCV treatment. This comprised $22 \%$ of all patients undergoing upper GIT endoscopy over that year. There was a fourfold rise in percentage of patients undergoing endoscopy for sofosbuvir-based therapy in this year (22\%) when compared to assessment for Interferon/Ribavirin combination therapy (5\%) in the previous year. A total of 331 patients had no esophageal or gastric varices. Small sized (grade I), medium sized (grade II) and large sized (grade III) varices were reported in 301,188 and 293 patients respectively. Thirty patients $(2.6 \%)$ had gastric varices. The prevalence of varices was higher in Child $B$ in comparison to Child A (statistically not significant, $p=0.243$ ). Conclusion: Screening endoscopy for early cirrhotic patients awaiting oral anti-HCV therapy is valid.

\section{Keywords}

Upper Gastro-Intestinal Endoscopy, HCV, Cirrhosis, Sofosbuvir, Esophageal Varices, Gastric Varices, Direct Acting Antivirals

\footnotetext{
${ }^{*}$ Corresponding author.
}

How to cite this paper: Mohamed, S.Y., Gaballah, B.A. and Emara, M.H. (2015) Validity of Upper Gastro-Intestinal Endoscopic Screening in HCV Cirrhotic Patients Awaiting Antiviral Therapy. Open Journal of Gastroenterology, 5, 191-199. 


\section{Introduction}

Hepatitis C virus (HCV) infection is a major public health burden in Egypt, where the highest prevalence rate of HCV in the world exists. Estimates for prevalence are based upon data reported from the 2008 Egypt Demographic and Health Survey, a nationally representative sample that included participants from all major Egyptian regions, where anti-HCV antibodies prevalence was $14.7 \%$, and viraemia was $9.8 \%$ [1]. The natural history of chronic HCV infection involves the development of liver cirrhosis in a large proportion of patients which may eventually progress to liver cell failure and hepatocellular carcinoma [2].

At least two thirds of cirrhotic patients will develop esophageal varices during their lifetime. Severe upper gastrointestinal bleeding as a complication of portal hypertension develops in about 30\% - $40 \%$ of cirrhotics. Despite the significant improvements in the early diagnosis and treatment of esophagogastric variceal hemorrhage, the mortality rate of the first variceal hemorrhage remains high (20\% - 35\%). Preventing first variceal hemorrhage offers the promise of reducing mortality, morbidity, and associated health care costs [3] [4]. Although many noninvasive parameters including routine liver biochemistry, esophageal wall thickness, and splenic diameter/platelet count ratio, APRI, Fibroscan of the spleen....etc. [5] [6], where tried to predict the presence of varices, esophagogastroduodenoscopy (EGD) remains the gold standard in the diagnosis of varices and it also offers therapeutic potentials [7].

Before 2006, Egypt did not have a comprehensive national program for control of viral hepatitis. HCV treatment program started with strong governmental support with the hope of eventual HCV eradication in Egypt. All eligible patients were treated with pegylated interferon/ribavirin (PEGIFN-RBV), with almost 100\% of the patients receiving therapy for free [8]. Viral hepatitis treatment centers opened nation-wide for application of PEGIFN-RBV therapy since 2007.

A number of new treatment options have become available, with much higher efficacy (cure rates $>90 \%$ ) compared to the previous standard of care PEGIFN-RBV [9]. Sofosbuvir was the first direct antiviral agent (DAA) introduced in Egypt, with large scale treatment starting in October 2014.

Priority of treatment in Egypt's HCV treatment program with new DAAs therapies is directed towards patients with stage III and stage IV hepatic fibrosis. No differentiation in treatment priority was granted based on previous treatment experience. Upper GIT endoscopy was mandatory according to the national Egyptian guidelines in the following circumstances: histologic evidence of cirrhosis by liver biopsy, fibroscan score $>19.2 \mathrm{k} \cdot \mathrm{pa}$ and Platelet count $<100,000 \times 10^{3} / \mathrm{dl}$. Exclusion criteria included a Child-Pugh class $\mathrm{C}$ with score $\geq 9$, presence of ascites (except after control), patients with HCC except after successful radical intervention (4 months after resection or successful local ablation) evident by triphasic CT and presence of large risky esophageal varices (except after prophylactic treatment) [10].

This study aimed at estimating the prevalence of varices among those patients and judging the validity of the national recommendation of upper GIT endoscopic screening before enrollment in HCV antiviral therapy and consequently assesses the work load on the endoscopic units met by this recommendation.

\section{Patients and Methods}

This study is a prospective study including patients evaluated at endoscopy units, Zagazig University Hospitals between January $1^{\text {st }}$ and December $31^{\text {st }} 2014$ for enrollment in HCV therapy. Patients were referred to our endoscopy units from HCV treatment clinics of the National Committee for Prevention and Control of viral hepatitis centers.

\subsection{Inclusion Criteria}

Patients with these criteria were enrolled in the study: patients with confirmed HCV infection, patients with age of 18 years and older, patients of both sexes.

All patients referred for preparatory upper GIT endoscopy were enrolled in the study. Clinical evaluation and routine biochemical records (including complete liver function tests) were reviewed at enrollment. Data were extracted from the patients themselves and the attached medical records with special emphasis on medical history, demographics, laboratory data, ultrasonic findings and sometimes clinical examination. Patients were classified into group A; patients screened for HCV treatment with Child Pugh class A and group B; patients screened 
for HCV treatment with Child Pugh class B.

\subsection{Endoscopic Examinations}

Endoscopic findings of all patients presented to our endoscopy units during the study period were analyzed including other cirrhotic patients on regular endoscopic follow up. Upper GIT Endoscopy was performed under light sedation in the left lateral position using midazolam in all cases. During the course of the endoscopy, a complete evaluation of the esophagus, stomach and duodenum was carried out, and the presence of esophageal and gastric varices was evaluated and recorded.

Endoscopic grading of esophageal varices was carried out after the Italian liver cirrhosis project, in which varices are graded according to the proportion of the radius of the esophagus occupied by the largest varix present at full insufflations of the esophagus [4]. Grade1: Varices that collapse to inflation of the esophagus with air. Grade 2: varices between grades 1 and 3. Grade 3: varices which are large enough to occlude the lumen.

Endoscopic classification of gastric varices was carried out after Sarin's Classification [7], which classifies gastric varices into: Type 1 gastroesophageal varices (GOV 1); are typically a continuation of esophageal varices into the lesser curvature. Type 2 gastroesophageal varices (GOV 2); when the esophageal and fundal varices are present in continuity over the cardia. Type 1 isolated gastric varices (IGV 1); are usually isolated gastric fundal varices. Type 2 isolated gastric varices (IGV 2); are gastric varices at ectopic sites in the stomach outside the cardio-fundal region or the first part of the duodenum.

\subsection{Ethical Issues}

The study protocol was approved by the Institutional Review Board of the Faculty of Medicine, Zagazig University, Egypt. All patients or patients' first-degree relatives provided written informed consent to participate in the study and to perform all relevant investigations including the endoscopy.

\subsection{Statistical Analysis}

All statistics were performed using SPSS 22.0 for windows (SPSS Inc., Chicago, IL, USA). Numbers and percentages were used for qualitative data while mean \pm standard deviation (SD) was used for quantitative ones. Percent of categorical variables were compared using the Pearson's Chi-square $\left(\chi^{2}\right)$ test. All tests were two sided, p-value $<0.05$ was considered significant.

\section{Results}

\subsection{Demographics of the Studied Patients}

During the study period a total of 5195 patients were endoscoped, of them 1143 patients performed upper GIT endoscopy in preparation for HCV treatment. Demographics of the 1143 studied patients are shown in Table 1. The laboratory results presented here assessed within two weeks prior to endoscopy. Patients with Child A (group A) were 364 patients, while patients with Child class B (group B) were 779 patients. There were no significant differences in the demographics between both groups. Most of our cases were males (63\%), and minority were already receiving prophylactic measures for portal hypertension (12.2\%), and of them $5.77 \%$ had mild ascites.

\subsection{Workload on Endoscopy Units}

A total of 1143 patients sought upper GIT endoscopy at our units in preparation for HCV treatment. This comprised 22\% of all patients undergoing upper GIT endoscopy over the study year. There was a fourfold rise in percentage of patients undergoing endoscopy for HCV therapy in this year (22\%) after starting enrollment for sofosbuvir-based therapy when compared to assessment for PEGIFN-RBV combination therapy (5\%) in the previous year. When the work on endoscopy units is compared in the two successive years 2013 and 2014, it becomes obvious that there is increase in the rate of endoscopy by $20.4 \%$, when specifically the load due to preparation for therapy is compared, there was a marked increase in the number of patients assessed for oral therapy when compared to PEGIFN-RBV (Table 2). During the second half of 2014 there was a noticeable rise in the frequency of endoscopies due to increase in the numbers of assessments (Figure 1). 
Table 1. Characteristics of the patients undergoing preparatory GIT endoscopy.

\begin{tabular}{lccc}
\hline & & Mean \pm SD & Range \\
\hline Age (years) & Male & $47 \pm 6.3$ & $20-70$ \\
Sex & Female & $63 \%$ & $17.4-37$ \\
BMI & & $37 \%$ & $6-15$ \\
Child Pugh score & YES & $24 \pm 2.7$ & $27.1-293$ \\
Ongoing treatment for PHT: & NO & $13 \pm 1$ & $16.8-284$ \\
ALT (IU/L) & & 140 & $2.2-5.1$ \\
AST (IU/L) & & $52.8 \pm 25.5$ & $53-540$ \\
Albumin (g/dL) & & $4.2 \pm 0.6$ & $0.5-4.7$ \\
Platelet count (×10 3 /dl) & & $233 \pm 168$ & $7-14$ \\
Total bilirubin (mg/dl) & No & $0.8 \pm 0.3$ & $10.6 \pm 1.4$ \\
Prothrombin time (seconds) & Mild & 66 & 1077 \\
Ascites & & & \\
\end{tabular}

BMI, body mass index, PHT, portal hypertension.

Table 2. Total case number over two years.

\begin{tabular}{cccc}
\hline Year & Preparatory & Others & Total \\
\hline 2013 & 292 & 4022 & 4314 \\
2014 & 1143 & 4052 & 5195 \\
\hline
\end{tabular}

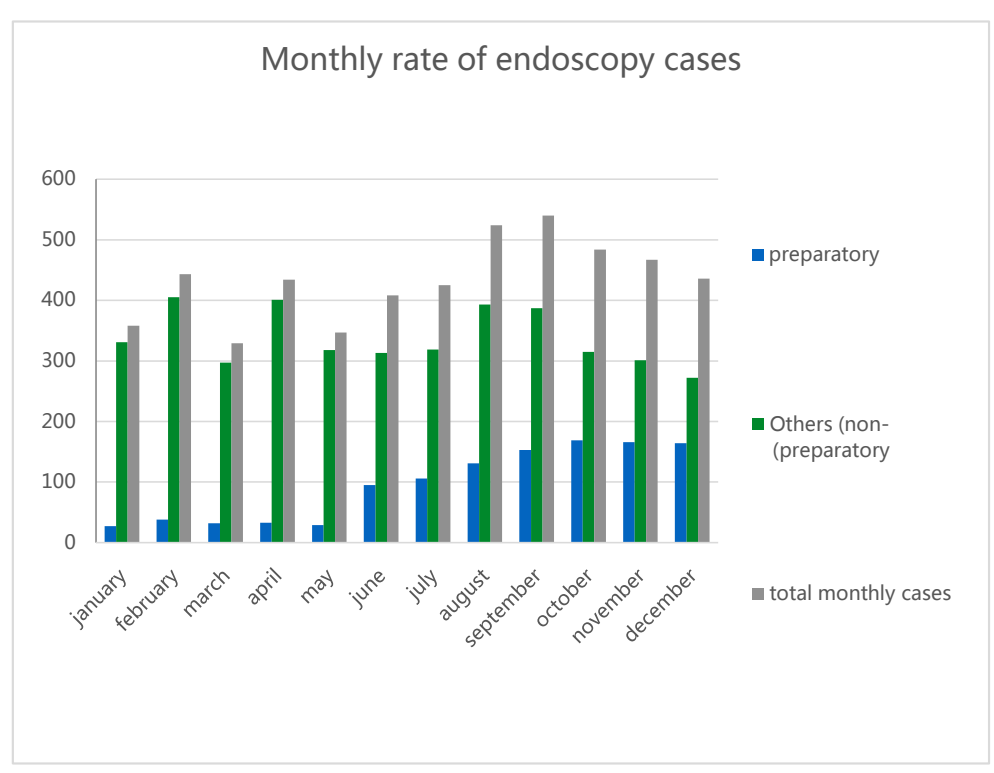

Figure 1. The rate of endoscopy per month during 2014. 


\subsection{Endoscopic Findings of Patients Awaiting DAAs}

When the data of the 1143 patient who performed endoscopy for oral HCV therapy were analyzed (Figure 2, Table 3) there were a total of 331 patients (28.95\%) who had no esophageal or gastric varices. Small sized esophageal varices (grade I) present in 301 (26.3\%) patients while medium sized varices (grade II) reported in 188 (16.4\%) patients and 293 (25.6\%) patients encountered in large sized varices (grade III). Only 2 patients (0.2\%) had isolated gastric varices without associated esophageal varices. Thirty patients (2.6\%) had gastric varices, of them 17 were GOV type 1, eleven were GOV type 2 and two were IGV type 1.

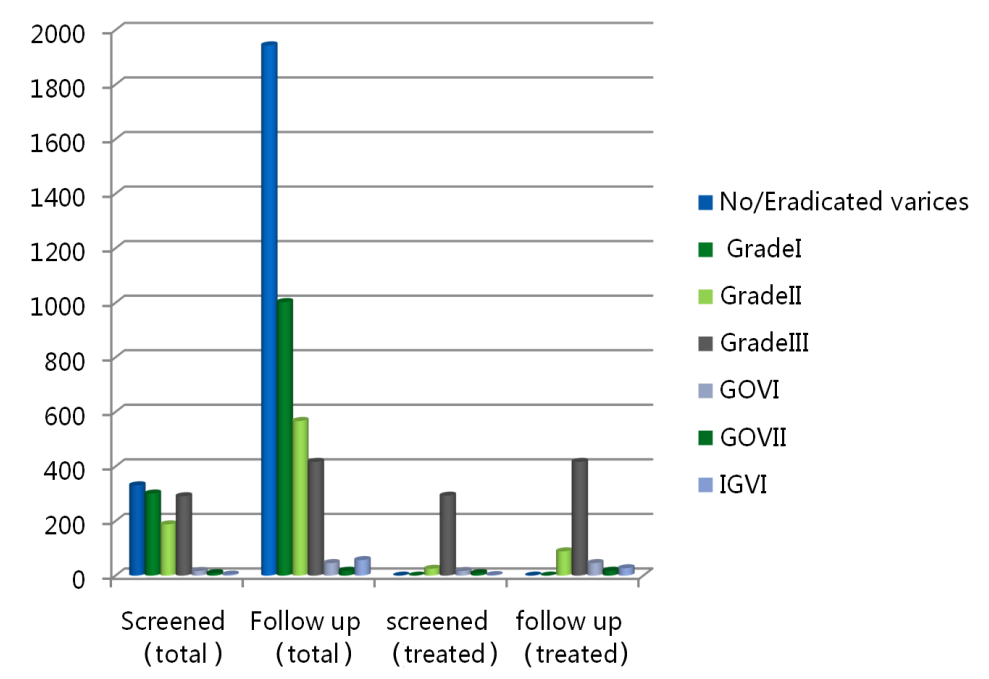

Figure 2. Major endoscopic findings of all patients during 2014. To the right of the figure, are the total number of patients screened (preparatory) and follow up (non-preparatory); while to the left side of the figure are the numbers of patients treated in each group.

Table 3. Endoscopic Findings of both groups.

\begin{tabular}{|c|c|c|c|}
\hline & $\begin{array}{c}\text { Child A } \\
(\mathrm{N}=364)\end{array}$ & $\begin{array}{c}\text { Child B } \\
(\mathrm{N}=779)\end{array}$ & p-value $e^{\S}$ \\
\hline \multicolumn{4}{|l|}{ Varices } \\
\hline Absent & $114(31.3 \%)$ & $217(27.9 \%)$ & \multirow{2}{*}{$\begin{array}{c}0.243 \\
\text { (NS) }\end{array}$} \\
\hline Present & $250(68.7 \%)$ & $562(72.1 \%)$ & \\
\hline \multicolumn{4}{|l|}{ Site } \\
\hline Esophageal varices & $240(96 \%)$ & $542(96.4 \%)$ & \multirow{3}{*}{$\begin{array}{c}0.123 \\
\text { (NS) }\end{array}$} \\
\hline Gastroesophageal varices & $10(4 \%)$ & $18(3.1 \%)$ & \\
\hline Lone Fundal varices (IGV type 1) & $0(0 \%)$ & $2(0.6 \%)$ & \\
\hline \multicolumn{4}{|l|}{ Esophageal Varices } \\
\hline Grade I & $100(40 \%)$ & $201(35.7 \%)$ & \multirow{3}{*}{$\begin{array}{c}0.250 \\
\text { (NS) }\end{array}$} \\
\hline Grade II & $50(20 \%)$ & $138(24.5 \%)$ & \\
\hline Grade III & $90(36 \%)$ & $203(36.1 \%)$ & \\
\hline \multicolumn{4}{|l|}{ Gastric varices } \\
\hline GOV type I & $5(2 \%)$ & $12(2.1 \%)$ & \multirow{2}{*}{$\begin{array}{l}0.689 \\
\text { (NS) }\end{array}$} \\
\hline GOV type II & $5(2 \%)$ & $6(1.06 \%)$ & \\
\hline
\end{tabular}

$\mathrm{N}=$ Total number of patients, GOV = gastroesophageal varices, $\mathrm{IGV}=$ isolated gastric varices, ${ }^{\S}$ Chi-square test; $\mathrm{p}<0.05$ is significant. 
Endotherapy (band ligation and/or gastric variceal injection) performed in 346 (30.3\%) patients, of them 318 had esophageal and 26 with gastroesophageal varices and only two had fundal varices without associated esophageal varices. Endoscopic band ligation was done using a multi-ligator device containing 7 rubber bands (LIGASHOOT) while fundal injection was performed using tissue adhesive glue (iso Amyl-2cyanoacrylate) (Amacrylate, Concord Drugs LTD, India).

Patients with fundal varices were treated using the glue injection. Also all patients with grade III varices were banded and only 25 cases with grade II varices (are those who showed risky signs during endoscopy i.e. cherry red spots and in whom the risk of variceal rupture exists) and those associated with GOV were banded. The remaining patients with non-risky varices scheduled for endoscopic follow up.

When the 1143 screened patients were compared with patients on regular endoscopic follow up who were examined in our endoscopy theaters during the study period, most of the patients in regular follow up had their varices eradicated (48\%) or had non-risky grade I or II $(38.7 \%)$ varices because they received multiple sessions of sclerotherapy and/or band ligation while screened patients had more risky varices (27.6\%) than follow up patients $(12.7 \%)(\mathrm{p}<0.001)$ and that is why more percentage of patients in the screened group received endoscopic management. The rate of gastric varices was comparable in both groups, however, all screened patient (100\%) received endoscopic management for their gastric varices whereas, not all patients (2.2\%) of follow up received treatment for IGVs, and that is because many of them had serpiginous thick walled veins that were scheduled for regular follow up (Figure 2).

When both groups are compared (Table 3), patients in group B had more esophageal and gastric varices than those of group A with numbers of 250 (68.7\%) and 562 (72.1\%) respectively, although this difference was not statistically significant ( $\mathrm{p}$ value $=0.243$ ). Furthermore, the severity of liver disease defined by Child class B was associated with risky gastric varices; patients with Child B had more risky varices (grade II and III) than patients with Child A cirrhosis, however, the difference was not statistically significant (p value $=0.25$ ).

And the same is also applied to gastric varices, patients with Child B had more gastric varices than patients with Child A however, the difference was not statistically significant

\section{Discussion}

The availability of a highly effective therapy, coupled with increased diagnosis and treatment and marked reduction in the new cases, has the potential to eliminate hepatitis $C$ in Egypt within the next decades because viral hepatitis is arguably the most significant public health problem facing Egypt [11]. HCV prevalence rates in the general population are estimated at $10 \%$ to $15 \%$ in rural areas, with some age groups suffering from prevalence rates of up to 50\%. As additional DAAs are approved for clinical use, clinicians may face complex choices and treatment guidelines [12]-[14].

Newly introduced DAAs for HCV are expected to increase eligibility for treatment. Hepatologists will probably face challenges in prioritizing patients for treatment [15] [16], and criteria for receiving therapy will be reshaped in parallel with changing therapeutic landscape [17].

In our study, patients presenting for upper GIT endoscopy as preparation for HCV therapy had mostly borderline laboratory findings, including liver function tests. Total number of such patients increased in 2014 as compared to 2013. There was also a trend of increase in the second half of 2014. Both findings can be explained by the introduction of the new HCV therapies, with increased eligibility for treatment and the priority criteria for enrollment in the national treatment program when compared with previous policy for IFN based regimens. Besides, the effect imparted by the mass media which encouraged patients for treatment armed by the high SVR rates in the preliminary studies.

Portal hypertension is a progressive complication of cirrhosis. The management of the patient with cirrhosis and portal hypertensive gastrointestinal bleeding depends on the phase of portal hypertension at which the patient is situated, from the patient with cirrhosis and portal hypertension who has not yet developed varices to the patient with acute variceal hemorrhage for whom the objective is to control the active episode and prevent rebleeding [3].

Gastric varices are less prevalent than esophageal varices and are present in 5\% - 33\% of patients with portal hypertension with a reported incidence of bleeding of about 25\% in 2 years, with a higher bleeding incidence for fundal varices [3]. In our series, gastric varices were reported in $2.6 \%$ and $3 \%$ of screened and follow up patients respectively. 
Our figures are less than the rates reported previously from the Egyptian literature that ranged from $12.5 \%$ to $13.3 \%$, and that is because our patients, in one hand were none bleeders and in the other hand were at earlier stages of cirrhosis when compared to patients with acute variceal hemorrhage including patients with Child C cirrhosis and HCC in the previous studies [18] [19].

A substantial number of screened patients (68.4\%) had esophageal and gastric varices mostly detected for the first time. A small proportion of those patients had history of previous endoscopy with medical treatment for portal hypertension. Endotherapy in the form of band ligation for esophageal varices and variceal injection for gastric varices was performed. The decision for endoscopic intervention for our cases coincided with the current guidelines [20]. In fact, endoscopic treatment of grade III varices and grade II varices with risky signs will reduce the frequency of $1^{\text {st }}$ variceal bleeding and that is why we endoscopically treated these patients.

Detection of such large number of patients with varices points to a large gap in our follow-up strategies in HCV-positive patients. However, detection of such large number of patients at earlier stages will shift management of varices from bleeding to prophylactic measures which probably may improve the general wellbeing and survival in HCV cirrhotic patients.

The presence of varices correlates with the severity of liver disease; while in the previous international studies only $40 \%$ of Child A patients had varices; about $85 \%$ of Child C patients had varices [4]. In the current study about $65.9 \%$ and $69.5 \%$ of screened patients with Child A and B had varices respectively. These figures are consistent with similar higher rates reported from the Egyptian literature [21] [22], and can be explained by the noncompliance of cirrhotic patients with the scheduled endoscopic screening in cirrhotics [20] and the bad reputation of IFN based therapies, were a large proportion of Egyptian patients canceled evaluation for IFN based therapies and were waiting the oral drugs. However, this study confirmed parallel increase [4] in the severity of varices (represented with increased grade) with advanced liver disease. This was noticeable by the higher prevalence of risky varices (grade II and III) in Child B patients compared to Child A patients, although the difference was not statically significant $(\mathrm{p}=0.25)$.

The observable trend for increased numbers of subjects requiring endoscopic evaluation prior to HCV treatment has many implications. First of all, workload is increasing on endoscopy units which are already loaded with many patients of portal hypertension and varices due to the high prevalence of chronic liver diseases in Egypt [18] [19] [23], and this is obvious by the four fold rise in pre-treatment endoscopic assessments and overall endoscopic examinations during 2014 in comparison to 2013. Second; more cases eligible for endotherapy are detected earlier with shifting of workload from advanced to earlier stages of CLD. A program for follow-up of endoscopically-detected early cases of varices should be established parallel to HCV treatment program in line with established guidelines. This will ultimately reduce the frequency and complications of variceal bleeding. Also, the effect of newer therapies on portal hypertension and its complications should be noted, yet recent report from Austria showed potential beneficial effect of interferon free regimens for HCV cirrhotic liver on portal hypertension with improved liver stiffness, platelet count, serum albumin and bilirubin levels, as well as prothrombin time [24].

Increased eligibility for therapy will generally increase the burden on national resources. This will probably lead to some prioritization strategy, which may be included in the enrollment criteria for therapy. Presence of varices together with other parameters points to advanced CLD and hence gives priority for initiation of oral HCV therapy, particularly with shortage of liver transplantation in Egypt. However, due to the short period from its introduction till now, our understanding of its effects on the course of chronic liver disease and portal hypertension together with the side effects profile is not yet clear.

This study had some limitations. The first of all, it is a single center study. The second is the lack of follow up of patients who received new oral therapies and studying its effect on the natural history of varices.

According to the results of this study screening endoscopy for early cirrhotic patients awaiting oral anti-HCV therapy is valid, because a substantial proportion of these patients had varices, and it seems that varices are higher in patients with Child B cirrhosis when compared with patients with Child A cirrhosis. Endoscopic work burden is expected to rise with increased demand on newer oral regimens. More cases eligible for endotherapy are detected earlier with shifting of workload from advanced to earlier stages of CLD.

\section{Conclusion}

In conclusion, screening endoscopy for early cirrhotic patients awaiting oral anti-HCV therapy is valid. Endos- 
copic work burden is expected to rise with increased demand on newer directly acting antiviral regimens.

\section{References}

[1] El-Zanaty, F. and Way, A. (2009) Egypt Demographic and Health Survey. Ministry of Health and Population, Cairo.

[2] Farci, P., Alter, H.J., Wong, D., Miller, R.H., Shih, J.W., Jett, B., et al. (1991) A Long-Term Study of Hepatitis C Virus Replication in Non-A, Non-B Hepatitis. New England Journal of Medicine, 325, 98-104. http://dx.doi.org/10.1056/NEJM199107113250205

[3] Poza Cordon, J., Froilan Torres, C., Burgos García, A., Gea Rodriguez, F. and Suárez de Parga, J.M. (2012) Endoscopic Management of Esophageal Varices. World Journal of Gastrointestinal Endoscopy, 4, 312-322. http://dx.doi.org/10.4253/wjge.v4.i7.312

[4] Italian Liver Cirrhosis Project (1987) Reliability of Endoscopy in the Assessment of Variceal Features. Journal of Hepatology, 4, 93-98. http://dx.doi.org/10.1016/S0168-8278(87)80015-6

[5] Berzigotti, A., Gilabert, R., Abraldes, J.G., Nicolau, C., Bru, C., Bosch, J., et al. (2008) Noninvasive Prediction of Clinically Significant Portal Hypertension and Esophageal Varices in Patients with Compensated Liver Cirrhosis. American Journal of Gastroenterology, 103, 1159-1167. http://dx.doi.org/10.1111/j.1572-0241.2008.01826.x

[6] Berzigotti, A., Seijo, S., Arena, U., Abraldes, J.G., Vizzutti, F., García-Pagán, J.C., et al. (2013) Elastography, Spleen Size, and Platelet Count Identify Portal Hypertension in Patients with Compensated Cirrhosis. Gastroenterology, 144, 102-111. http://dx.doi.org/10.1053/j.gastro.2012.10.001

[7] Sarin, S.K., Lahoti, D., Saxena, S.P., Murthy, N.S. and Makwana, U.K. (1992) Prevalence, Classification and Natural History of Gastric Varices: A Long-Term Follow-Up Study in 568 Portal Hypertension Patients. Hepatology, 16, 13431349. http://dx.doi.org/10.1002/hep.1840160607

[8] Doss, W., Mohamed, M.K., Esmat, G., El Sayed, M., Fontanet, A., Cooper, S., et al. (2008) Egyptian National Control Strategy for Viral Hepatitis 2008-2012. Arab Republic of Egypt, Ministry of Health and Population, National Committee for the Control of Viral Hepatitis. http://www.hepnile.org/images/stories/doc/NSP_10_April_2008_final2.pdf

[9] Afdhal, N.H., Zeuzem, S., Schooley, R.T., Thomas, D.L., Ward, J.W., Litwin, A.H., et al. (2013) The New Paradigm of Hepatitis C Therapy: Integration of Oral Therapies into Best Practices. Journal of Viral Hepatitis, 20, 745-760. http://dx.doi.org/10.1111/jvh.12173

[10] Doss, W. National Committee for the Control of Viral Hepatitis: Hope for Eradication. http://www.liver-eg.org/assets/wahid-national-committee-for-control-treatment.pdf

[11] Estes, C., Abdel-Kareem, M., Abdel-Razek, W., Abdel-Sameea, E., Abuzeid, M., Gomaa, A., et al. (2015) Economic Burden of Hepatitis C in Egypt: The Future Impact of Highly Effective Therapies. Alimentary Pharmacology \& Therapeutics, 42, 696-706. http://dx.doi.org/10.1111/apt.13316

[12] Waked, I., Doss, W., El-Sayed, M.H., Estes, C., Razavi, H., Shiha, G., et al. (2014) The Current and Future Disease Burden of Chronic Hepatitis C Virus Infection in Egypt. Arab Journal of Gastroenterology, 15, 45-52. http://dx.doi.org/10.1016/j.ajg.2014.04.003

[13] Emam, M., Radwan, M., Refaey, M., Shaheen, N., Abd-ElMoez, S. and Gerges, M. (2015) Prevalence of Hepatitis C Virus in an Elderly Population in Rural Areas of Sharkia Governorate, Egypt. Egyptian Liver Journal, 5, 29-33. http://dx.doi.org/10.1097/01.ELX.0000463678.53334.c7

[14] Hepatitis, C. (2007) WHO Fact Sheet No. 164. 45-52. http://www.who.int/mediacentre/factsheets/fs164/en/

[15] Borgia, S.M. and Rowaiye, A. (2015) Increased Eligibility for Treatment of Chronic Hepatitis C Infection with Shortened Duration of Therapy: Implications for Access to Care and Elimination Strategies in Canada. Canadian Journal of Gastroenterology \& Hepatology, 29, 125-129.

[16] Hayes, C.N. and Chayama, K. (2015) Emerging Treatments for Chronic Hepatitis C. Journal of the Formosan Medical Association, 114, 204-215. http://dx.doi.org/10.1016/j.jfma.2014.09.001

[17] European Association for the Study of the Liver (2015) EASL Recommendations on Treatment of Hepatitis C 2015. Journal of Hepatology, 63, 199-236. http://dx.doi.org/10.1016/j.jhep.2015.03.025

[18] Elwakil, R., Reda, M.A., Abdelhakam, S.M., Ghoraba, D.M. and Ibrahim, W.A. (2011) Causes and Outcome of Upper Gastrointestinal Bleeding in Emergency Endoscopy Unit of Ain Shams University Hospital. Journal of the Egyptian Society of Parasitology, 41, 455-467.

[19] Abdel-Wahab, M., el-Ebidy, G., Gad el-Hak, N., Abou-Elenin, A., Abou-Zid, M., el-Ghawalby, N., et al. (1999) Fundal Varices: Problem and Management. Hepato-Gastroenterology, 46, 849-854.

[20] Garcia-Tsao, G., Sanyal, A.J., Grace, N.D., Carey, W., Practice Guidelines Committee of the American Association for the Study of Liver Diseases, Practice Parameters Committee of the American College of Gastroenterology (2007) AASLD Practice Guidelines: Prevention and Management of Gastroesophageal Varices and Variceal Hemorrhage in 
Cirrhosis. Hepatology, 46, 922-938.

[21] El Naggar, A.A., Gomaa, M.S. and Fawzy, M.M. (2012) Nonendoscopic Predictors of Large Esophageal Varices. The Egyptian Journal of Internal Medicine, 24, 97-99.

[22] Hussein, H.A., Tamer, W.M. and Khalaf, M.S. (2015) AST/ALT Ratio for Detection and Severity Classification of Esophageal Varices in Child A HCV Liver Cirrhosis in Egyptian Patients. Life Science Journal, 12, 12-17.

[23] Abdelaziz, A. and Yousif, M. (2014) Prevalence and Outcome of Bleeding Gastro-Esophageal Varices in Medical Intensive Care Unit at Zagazig University Hospitals, Egypt. Afro-Egyptian Journal of Infectious and Endemic Diseases, 4, 13-22.

[24] Mandorfer, M., Kozbial, K., Freissmuth, C., Schwabl, P., St€attermayer, A.F., Reiberger, T., et al. (2015) Interferon-Free Regimens for Chronic Hepatitis C Overcome the Effects of Portal Hypertension on Virological Responses. Alimentary Pharmacology \& Therapeutics, 42, 707-718. http://dx.doi.org/10.1111/apt.13315 